\title{
Validade e confiabilidade do Alcohol Use Disorders Identification Test (AUDIT) em estudantes de uma universidade brasileira
}

\author{
Validity and reliability of the Alcohol Use Disorders Identification Test (AUDIT) in \\ students of a Brazilian university
}

\section{Validez y confiabilidad del Alcohol Use Disorders Identification Test (AUDIT) en estudiantes de una universidad brasileña}

\author{
Kairon Pereira de Araújo Sousa ${ }^{1}$ ORCID 0000-0003-0779-343X \\ Emerson Diógenes de Medeiros ${ }^{2}$ ORCID 0000-0002-1407-3433 \\ Paloma Cavalcante Bezerra de Medeiros ${ }^{3}$ ORCID 0000-0002-5868-8333 \\ ${ }^{123}$ Universidade Federal do Piauí, Parnaíba. Brasil
}

Resumo: Esta pesquisa objetivou avaliar as propriedades psicométricas (validade e precisão) do Alcohol Use Disorders Identification Test (AUDIT) em estudantes de uma universidade pública brasileira. Participaram 406 estudantes universitários, com idade média de 23,31 anos $(D P=5,73)$, a maioria do sexo feminino $(68,2 \%)$, solteiros $(58,4 \%)$, com padrões de consumo de álcool de baixo risco $(74,4 \%)$, que responderam o AUDIT e questões sociodemográficas. Realizou-se análises descritivas, análises fatoriais confirmatórias $(\mathrm{AFC})$ e de consistência interna. Os resultados indicaram melhor ajuste do modelo trifatorial $\left[\chi^{2}(32)=0,89, p<, 001\right.$; TLI $=, 99$; CFI $=, 99$; RSMEA $=0,036$ $(\mathrm{IC} 90 \%=0,012-0,056)$; ECVI $=0,30]$ e adequada consistência interna $(\alpha=, 85 ; \Omega=, 89)$. Conclui-se que o instrumento demonstrou-se adequado teórica e metricamente, podendo ser utilizado em pesquisas que objetivam medir padrões de consumo de álcool em amostras semelhantes a aqui considerada.

Palavras-chave: AUDIT; consumo de álcool; precição; validade

Abstract: The objective of this research was to assess the psychometric properties (validity and precision) of the Alcohol Use Disorders Identification Test (AUDIT) in students of a Brazilian public university. A total of 406 university students participated, with an average age of 23.31 years $(S D=$ $5.73)$, most of them were women (68.2\%), single (58.4\%), with low risk alcohol consumption (74.4\%), who answered the AUDIT test and sociodemographic questions. Descriptive analyzes, confirmatory factor (CFA) and internal consistency analyzes were performed. The results indicated a better fit of the three-factor model $\left[\chi^{2}(32)=0.89, p<.001 ; \mathrm{TLI}=0.99 ; \mathrm{CFI}=0.99 ; \mathrm{RSMEA}=0.036(\mathrm{CI} 90 \%=0.012\right.$ - 0.056); ECVI $=0.30]$ and adequate internal consistency $(\alpha=.85 ; \Omega=.89)$. It is concluded that the instrument proved to be theoretically and metrically suitable, and it can be used in research aimed at assessing alcohol consumption patterns in samples similar to the one considered here.

Keywords: AUDIT; alcohol consumption; precision; validity 
Resumen: Esta investigación analizó las propiedades psicométricas (validez y precisión) del Alcohol Use Disorders Identification Test (AUDIT) en estudiantes de una universidad pública brasileña. Participaron 406 estudiantes universitarios, con una edad promedio de 23.31 años $(D E=5.73)$, la mayoría mujeres (68.2\%), solteras $(58,4 \%)$, con patrones de consumo de alcohol de bajo riesgo (74.4\%), que respondieron el AUDIT y un cuestionario sociodemográfico. Se realizaron análisis descriptivos, análisis factoriales confirmatorios (AFC) y de consistencia interna. Los resultados indicaron mejor ajuste del modelo trifactorial $\left[\chi^{2}(32)=0.89, p<.001 ; \mathrm{TLI}=.99 ; \mathrm{CFI}=.99 ; \mathrm{RSMEA}=0.036(\mathrm{IC} 90 \%=0.012-0.056)\right.$; ECVI= $0,30]$ y adecuada consistencia interna $(\alpha=.85 ; \Omega=.89)$. Se concluye que el instrumento se demostró adecuado teórica y métricamente, pudiendo ser utilizado en investigaciones que busquen medir patrones de consumo de alcohol en muestras semejantes a la aquí considerada.

Palabras clave: AUDIT; consumo de alcohol; precisión; validez

Recebido: 06/01/2019

Aceito: $20 / 07 / 2020$

Como citar:

Sousa, K.P.A., Medeiros, E.D., \& Bezerra de Medeiros, P.C. (2020). Validade e confiabilidade do Alcohol Use Disorders Identification Test (AUDIT) em estudantes de uma universidade brasileira. Ciencias Psicológicas, 14(2), e-2230. doi: https://doi.org/10.22235/cp.v14i2.2230

Correspondência: Emerson Diógenes de Medeiros. Universidade Federal do Piauí. Laboratório de Avaliação Psicológica do Delta - LABAP, CEP 64202-020, Parnaíba, PI - Brasil. E-mails: emersondiogenes@gmail.com; kaironpereira@hotmail.com; palomacbmedeiros@gmail.com

\section{Introdução}

O consumo de álcool é uma prática cultural incentivada em diversas sociedades do mundo (Silva et al., 2015). Seu uso permeia a vida das pessoas desde a mais tenra idade, vinculando-se como uma espécie de tradição, costume, código ou moral (Cabral, Farate, \& Duarte, 2007), expresso por virtudes simbólicas marcadas por mitos difundidos em conceitos que são responsáveis por manter o hábito de consumi-lo (Pinto, 1999).

A bebida alcoólica tem sido amplamente utilizada, tanto por ser classificada como uma droga lícita quanto por suas características psicoativas que promovem a sociabilidade e a integração entre as pessoas, sendo seu uso estimulado em diferentes ocasiões (Damacena et al., 2016). Contudo, apesar do consumo do álcool ser aceito e incentivado, a substância tem sido relacionada com frequência a diversos danos psicossociais, como doenças (Organização Mundial da Saúde [OMS], 2014), comportamentos delituosos (Feijão et al., 2012) e acidentes de trânsito (Mello Jorge \& Adura, 2013), provocando custos econômicos em todo o mundo (Rathod, Nadkarni, Bhana, \& Shidhaye, 2015).

No que se refere, especificamente, ao contexto acadêmico, o álcool é apontado como o principal problema de saúde pública na população universitária (Davoren, Dahly, Shiely, \& Perry, 2017). Em pesquisas prévias, tem sido documentada uma prevalência maior de uso de bebidas alcoólicas por esse público, em comparação à população geral ou mesmo entre estudantes de outros níveis educacionais (Baumgarten, Gomes, \& Fonseca, 2012). Os estudantes universitários são mais suscetíveis porque desenvolvem padrões típicos de uso, implicando em um beber problemático, forma de consumo que provoca danos individuais e sociais, que é reforçado por ações específicas entre este grupo (Peuker, Fogaça, \& Bizarro, 2006). 
Na literatura, o consumo excessivo de bebidas etílicas por universitários aparece associado a ações impulsivas, envolvimento em brigas, condução veicular sob efeito de álcool, baixo rendimento nas avaliações acadêmicas, etc. (Cardoso, Barbosa, Costa, Vieira, \& Caldeira, 2015). Tendo isso em conta, o padrão de uso de álcool entre esses jovens tem merecido especial atenção em investigações científicas acerca do tema, uma vez que os custos e consequências dessa prática são consideráveis em diferentes aspectos: sociais, econômicos e individuais (Baumgarten et al., 2012; Rocha, Lopes, Martelli, Lima, \& Martelli, 2011).

Dados os diferentes padrões de ingestão de álcool entre estudantes universitários, evidencia-se a necessidade de se contar com um instrumento de medida válido que possibilite detectá-los precocemente, objetivando a elaboração de medidas preventivas direcionadas a esse público. Dentre os testes empregados na literatura para a mensuração do construto, tem merecido especial atenção o Alcohol Use Disorder Identification Test (AUDIT).

O AUDIT foi desenvolvido pela OMS no final da década de 1980, projetado para uso na Atenção Primária à Saúde (APS), a partir de um projeto envolvendo um grupo internacional de pesquisadores que iniciou um estudo transnacional em seis países - Noruega, Austrália, Quênia, Bulgária, México e Estados Unidos da América - (Babor, Higgins-Biddle, Saunders, \& Monteiro, 2001), com o objetivo de tornar o instrumento adquado para uso em diferentes realidades socioculturais e econômicas (Méndez, 1999). Atualmente, o teste é o mais utilizado no mundo todo para a triagem do uso de álcool (García, Novalbos, Martínez, \& O'Ferrall, 2016), e apresenta como vantagem, em relação a outros instrumentos que medem o mesmo construto, o fato de permitir o rastreio de diferentes padrões de consumo, não se concentrando apenas na dependência (Babor et al., 2001).

Durante mais de duas décadas, o instrumento foi construído e avaliado demonstrando ser uma medida precisa para a avaliação de problemas decorrentes do uso álcool. Entretanto, apesar de ter apresentado qualidades psicométricas adequadas, atestando-o como uma medida de natureza transcultural, após sua publicação, seus criadores apontaram a necessidade de pesquisas adicionais de validação do teste (Babor et al., 2001).

Estudos prévios que avaliaram a sua estrutura fatorial, por meio de técnicas de análise fatorial exploratória e confirmatória, identificaram modelos de um (Noorbakhsh et al., 2018), dois (Von-der-Pahlen et al., 2008) e três fatores (García et al., 2016). Entretanto, segundo MenesesGaya (2011), embora haja documentação em estudos de estruturas com itens saturando em um único fator, estas aparecem registradas em menor frequência comparadas aos modelos com dois e três fatores.

Conforme a autora supracitada, na atualidade, verifica-se um número maior de pesquisas sustentando um modelo bifatorial (Meneses-Gaya, 2011). De fato, as investigações sobre a dimensionalidade do AUDIT, tanto em âmbito internacional (Campos-Arias, Villamil-Vargas, \& Herazo, 2013; Seguel Palma, Santander Manríquez, \& Alexandre Barriga, 2013) quanto no contexto brasileiro (Lima et al., 2005), têm dado suporte majoritariamente, a partir de análises estatísticas, a um instrumento bifatorial.

Uma pesquisa realizada com 1000 estudantes universitários de dois campi localizados na cidade de Bogotá, Colômbia, analisou as propriedades psicométricas (validade de construto e confiabilidade) do AUDIT, através de análise fatorial confirmatória, identificando estrutura de duas dimensões (Castañeda, Munévar, Lara, \& Villa, 2016). Em outro estudo desenvolvido com 173 adolescentes, os pesquisadores examinaram a estrutura original de três domínios para uma versão modificada do AUDIT. Os resultados apontaram que a estrutura bidimensinal (consumo e dependência/conseqüências) proporcionou o melhor ajuste aos dados (Chung, Colby, Barnett, \& Monti, 2002).

No Brasil, estudos realizados nas regiões sudeste e nordeste do país apoiaram a estrutura composta por dois fatores do AUDIT. O primeiro foi realizado com amostra de 530 pacientes de um Pronto Socorro e um Centro de Atenção Psicossocial Álcool e Drogas - CAPS AD, em São 
Paulo. Executando-se análise fatorial exploratória (AFE), foram encontrados dois fatores. O fator 1, relacionado ao padrão de consumo de álcool, foi composto pelos itens um, dois, três e dez; o fator 2, associado aos problemas e consequências ocasinadas pelo consumo de álcool, reuniu os itens de quatro a nove (Meneses-Gaya, 2011).

$\mathrm{Na}$ segunda pesquisa, realizada em Fortaleza - CE, participaram 547 estudantes universitários, empregando técnica de análise fatorial exploratória. $\mathrm{O}$ modelo bifatorial mostrouse adequado, com o primeiro fator, foi denominado de Frequência e consequências adversas do consumo, sendo formado por sete itens (um, dois, três, sete, oito, nove e dez) e o segundo fator, nomeado como Dependência, reunindo os itens: quatro, cinco e seis (Santos et al., 2012).

Estes achados sustentam um modelo de dois fatores que contradiz o desenho original tridimensional do AUDIT, como um instrumento de triagem em relação ao consumo de álcool, proposto pela OMS (Babor et al., 2001). É preciso considerar, entretanto, como ressaltam Meneses-Gaya, Zuardi, Loureiro e Crippa (2009), que as análises fatoriais efetuadas com dados oriundos do instrumento podem ser afetadas por diferentes aspectos, a exemplo da amostra utilizada.

Apesar da relevância destas pesquisas, concordamos com Altmam e Bland (1994) de que um instrumento só é valido no contexto em que é validado. Assim, considerando que o Brasil é um país com dimensões continentais, com contextos culturais diversos (Maciel, 2017), a realização de novos estudos, com o intuito de avaliar a validade e dimensionalidade do AUDIT, parece plausível, principalmente tendo em conta que diferentes amostras podem produzir resultados distintos, por exemplo, do modelo original (Allen, Litten, Fertig, \& Babor, 1997).

Ademais, pesquisas adicionais sobre o instrumento são relevantes por possibilitar a comparação com os achados de outros estudos, contribuindo para o avanço da ciência. A partir do exposto, e considerando a inexistência de estudos de validação do AUDIT em amostra universitária no estado do Piauí, é que se procura realizar a avaliação das propriedades psicométricas deste instrumento para o contexto supracitado.

\section{Método}

\section{Participantes}

Participaram 406 estudantes universitários de uma Instituição de Ensino Superior (IES) pública, localizada no estado do Piauí, a maioria pertencente área das ciências biológicas $(27,6 \%)$, com idade variando de 18 a 58 anos $(M=23,31 ; D P=5,73)$, sexo feminino $(68,2 \%)$, solteiros $(58,4 \%)$, com renda média de $\mathrm{R} \$ 2.054,84$, equivalente a U\$S 384,44 , e de religião católica $(73,4 \%)$. Da totalidade dos participantes, $41,4 \%$ residem com os pais.

Quanto aos padrões de consumo de álcool da amostra selecionada a partir dos escores do AUDIT, verificou-se que $74,4 \%$ dos participantes encontram-se na Zona I (consumo de baixo risco), 20,7\% na Zona II (consumo de risco), 3,7\% na Zona III (consumo nocivo) e 1,2\% na Zona IV (provável dependência). Tratou-se de uma amostra não probabilística (acidental), fazendo parte universitários, maiores de idade ( $\geq 18$ anos), devidamente matriculado(a)s em um curso superior e que, aceitaram, de forma voluntária, colaborar com o estudo.

\section{Instrumentos}

- Alcohol Use Disorders Identification Test (AUDIT): esse instrumento foi desenvolvido pela OMS para avaliar padrões de consumo de álcool (Babor et al., 2001; Noorbakhsh et al., 2018), tendo sido validado para o português brasileiro por Figlie et al. (1997). O AUDIT é composto por dez itens que variam de 0 a 4 permitindo, assim, margem de pontuação de 0 a 40 . A partir da pontuação do participante, nessa escala de medida, é possível classificá-lo em quatro zonas 
(padrões) de consumo: baixo risco - 0 a 7 pontos; uso de risco - 8 a 15 pontos; uso nocivo - 16 a 19; e dependência - 20 a 40 pontos (Silva \& Tucci, 2014).

- Questionário sociodemográfico: composto por questões como idade, sexo, estado civil, renda e outras, objetivando caracterizar a amostra.

\section{Procedimento}

Inicialmente, entrou-se em contato com a direção da IES, local da pesquisa, com o intuito de se obter autorização para a aplicação de questionários com os estudantes que aceitassem colaborar, voluntariamente. Após a autorização dos dirigentes, adicionado a aprovação do projeto pelo Comitê de Ética em Pesquisa (CEP), da Universidade Federal do Piauí (Parecer no 2.400.755/2017), iniciou-se a coleta.

Os dados foram coletados em ambiente coletivo de sala de aula, com anuência dos professores, mediante agendamento prévio. Apesar da aplicação dos instrumentos ter ocorrido coletivamente, cada participante respondeu, de forma individual, aos questionários. Inicialmente eram apresentados os objetivos do estudo, apresentando esclarecimentos adicionais a respeito do anonimato, sigilo e confidencialidade dos dados. Enfatizou-se também que a participação no estudo era voluntária, sendo possível desistir a qualquer momento sem quaisquer consequências. Os participantes que concordaram em participar do estudo assinaram o Termo de Esclarecimento Livre e Esclarecido (TCLE).

A aplicação dos instrumentos de pesquisa foi realizada pelo pesquisador responsável e colaboradores devidamente treinados, que estiveram presentes durante todo o processo de coleta, para instruir os participantes acerca do preenchimento dos instrumentos e dirimir as possíveis dúvidas. Foram necessários, em média, 10 minutos para a conclusão dos questionários. Ressaltase que foram respeitadas todas as recomendações éticas em relação à pesquisa com seres humanos, conforme a Resolução no. 466/12 e 510/2016 do Conselho Nacional de Saúde.

\section{Análise dos dados}

Os dados foram analisados através do Software SPSS, versão 21, e R, na sua versão 3.5.1. Com o primeiro realizou-se o cálculo de estatísticas descritivas (medidas de tendência central e dispersão), visando caracterizar a amostra. Enquanto que por meio do R, foram realizadas Análises Fatoriais Confirmatórias (AFC), objetivando investigar a adequação do modelo teórico aos dados empíricos, ou seja, evidências de validade. Para tanto, contou-se com o pacote estatístico lavaan, e ainda com o Psych, para o cálculo da consistência interna (Alfa de Cronbach e Ômega de McDonald), cujos valores acima de ,70, tanto para o alfa quanto para o ômega, foram considerados aceitáveis (Gadermann, Guhn, \& Zumbo, 2012). Para avaliação dos modelos testados, uni, bi e trifatorial, os seguintes indicadores de ajustes foram tidos em conta (Hair, Black, Babin, Anderson, \& Tatham, 2009; Tabachnick \& Fidell, 2013):

(1) $\chi^{2}$ (Qui-quadrado). Indica a probabilidade de o modelo se ajustar aos dados, sendo recomendados valores baixos. Considerando que este indicador é sensível ao tamanho da amostra, sugere-se utilizar à sua razão em relação aos graus de liberdade do modelo $\left(\chi^{2} / g l\right)$, onde valores entre 2 e 3 são indicativos de um bom ajuste, admitindo-se até 5.

(2) Comparative Fit Index (CFI). É um índice adicional de ajuste do modelo, que serve para comparar com modelos alternativos. Seus valores variam de 0 a 1 (ajuste perfeito), aceitandose valores superiores a 0,90 como indicativo de um modelo ajustado.

(3) Tucker-Lewis Index (TLI). Este índice serve de comparação do modelo estimado com um modelo teórico nulo, propondo-se a estabelecer se todos os indicadores aparecem associados a um único fator latente. Valores superiores a 0,95 são indicativos de um ajuste robusto. 
(4) Root-Mean-Square Error of Approximation (RMSEA). O indicador, com intervalo de confiança de 90\% (IC90\%), serve para avaliar o quanto o modelo teórico se ajusta aos dados empíricos. Valores elevados indicam um modelo desajustado. Assim, recomenda-se valores próximos ou menores a 0,05 e 0,08, admitindo-se até 0,10 como um modelo aceitável.

Para comparação dos modelos alternativos e o de referência (três fatores), foram utilizados o teste de diferença entre os qui-quadrados $\left(\chi^{2}\right)$ e o graus de liberdade $(g l)$ de cada um deles $\left[\Delta \chi^{2}(g l)\right]$, considerado o mais ajustado aquele com menor valor de $\chi^{2}$, o Consistent Akaike information Criterion (CAIC) e o Expected Cross Validation Index (ECVI). Em relação a estes, valores mais baixos sugerem um modelo mais adequado.

\section{Resultados}

Com o intuito de reunir evidências psicométricas acerca do AUDIT no estado do Piauí, procurou-se verificar a adequação do modelo trifatorial proposto pela OMS. Para tanto, realizouse uma análise fatorial confirmatória (AFC), através do R e pacote Lavaan, adotando o estimador WLSMV (mínimos quadrados ponderados ajustados pela média e variância). Foram encontrados os seguintes resultados para os indicadores de ajustes deste modelo: $\chi^{2}(32)=0,89, p<, 001$; TLI= ,99; CFI= ,99; RSMEA=0,036 (IC90\%=0,012 - 0,056); ECVI=0,30.

Em seguida, visando identificar o modelo mais ajustado aos dados, testaram-se outras duas estruturas encontradas na literatura. Incialmente, procedeu-se uma AFC para o modelo bifatorial, que agrupou os domínios "Frequência e consequências adversas do consumo" e "Dependência". O modelo apresentou-se ligeiramente menos ajustado que o anteriormente testado, seus indicadores de ajuste foram $\chi^{2}(25)=2,13, p<, 001$; TLI $=, 98$; CFI= 99; RSMEA= 0,064 (IC90\%= 0,048-0,080); ECVI=0,36. Após testar o modelo de dois fatores, levou-se a cabo a análise da estrutura unifatorial, composta por uma única dimensão, que apresentou os seguintes resultados: $\chi^{2}(35)=2,01, p<, 001 ;$ TLI= ,98; CFI= 98; RSMEA = 0,069 (IC90\%=0,054 - 0,085); ECVI=0,39.

Os resultados obtidos, a partir das AFCs executadas com os diferentes modelos são sumarizados na Tabela 1. Ademais, buscou-se conhecer em que medida os modelos diferem entre si, estatisticamente. Para tanto, calculou-se a diferença entre o delta qui-quadrado e os graus de liberdade $\left[\Delta \chi^{2}(g l)\right]$ dos modelos propostos. O que permitiu avaliar diferenças estatisticamente significativas $(p \leq, 05)$ entre eles.

Tabela 1

Indicadores de ajustes dos modelos testados

\begin{tabular}{llllclll}
\hline Modelo & $\chi^{2}$ & GL & TLI & CFI & $\begin{array}{l}\text { RSMEA } \\
(\mathbf{C I 9 0 \% )}\end{array}$ & ECVI & $\Delta \boldsymbol{\chi}^{2} /(\mathbf{g l})$ \\
\hline Um & 70,60 & 35 & 0,98 & 0,98 & $\begin{array}{l}0,069 \\
(0,054-0,085)\end{array}$ & 0,39 & - \\
Dois & 53,2 & 25 & 0.98 & 0,99 & $\begin{array}{l}0,064 \\
(0,048-0,080)\end{array}$ & 0,36 & $17,4(10)^{*}$ \\
Três & 28,57 & 32 & 0,99 & 0,99 & $\begin{array}{l}0,036 \\
(0,012-0,056)\end{array}$ & 0,30 & $24,63(7)^{*}$ \\
\hline
\end{tabular}

Nota: $N$ (406); $\chi^{2}=$ qui-quadrado; $g 1$ = Graus de Liberdade; TLI = Tucker-Lewis Index; CFI = Comparative Fit Index; RMSEA = Root-Mean-Square Error Approximation, IC 90\% = Intervalo de Confiança de 90\%; ECVI = Expected Cross-Validation Index, CAIC $=$ Consistente Akaike Information Criterion; e $\Delta \chi^{2}(\mathrm{gl})=$ diferença entre os valores do qui-quadrado; $* p<, 01$. 
Tendo em conta os resultados previamente sumarizados na Tabela 1 , verifica-se que os modelos com três e dois fatores foram os que apresentaram melhores índices de ajustes em comparação à estrutura unifatorial. Contudo, considerando os indicadores de ajuste, observa-se que o modelo de três fatores se mostrou mais ajustado $\left(\chi^{2} / g l=0,89\right.$; TLI= ,99; CFI= ,99; RSMEA= 0,036). Ademais, o valor do ECVI, na estrutura trifatorial, é inferior aos apresentados nas demais, indicando ser um modelo mais parcimonioso. Ressalta-se, também, que todos os lambdas $(\lambda)$, de todos os modelos, foram estatisticamente significativos e diferentes de zero $(\lambda \neq 0 ; \mathrm{z}>1,96, p<$ ,05). A representação estrutural deste modelo, juntamente com os lambdas $(\lambda)$, é exposta na figura 1.

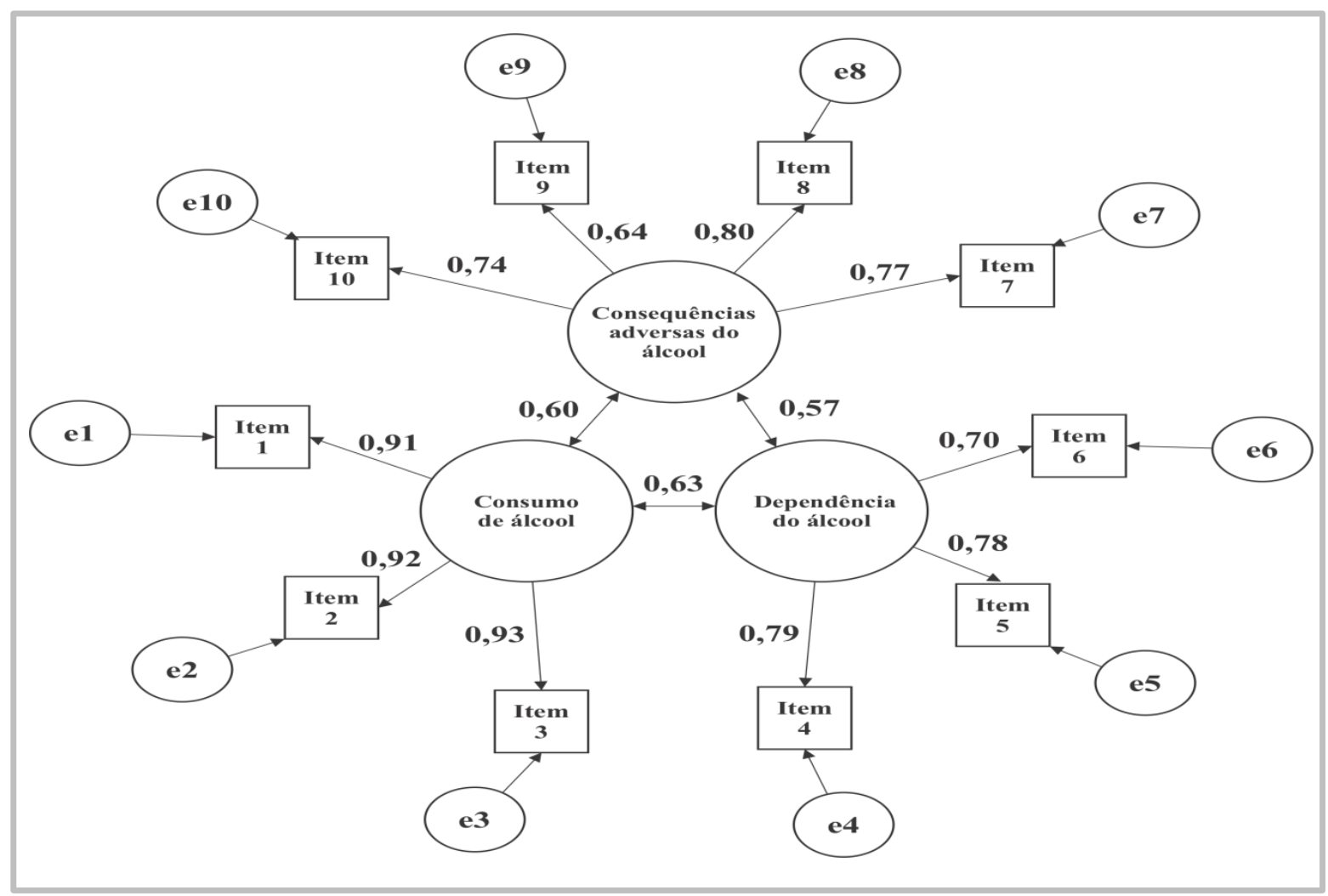

Figura 1.

Modelo Trifatorial do AUDIT

Evidências do modelo fatorial asseguradas, partiu-se para a próxima etapa, avaliar a fidedignidade do modelo mais adequado. Para tanto, efetuou-se o cálculo do alfa de Cronbach (com correlações policóricas) e ômega de McDonald, objetivando avaliar a sua consistência interna. Os resultados indicam adequada consistência interna para todos os fatores, a saber: Consumo de álcool $(\alpha=, 94 ; \Omega=, 88)$, Dependência do consumo de álcool $(\alpha=, 81 ; \Omega=, 68) \mathrm{e}$ Consequências adversas do consumo de álcool $(\alpha=, 82 ; \Omega=, 66)$. Adicionalmente, quando tratada como unidimensional, os valores de alfa e ômega foram de, 85 e, 89 , respectivamente.

\section{Discussão}

Este estudo averiguou a adequação do AUDIT no contexto Piauiense. Para tanto, foram testados diferentes modelos estruturais (uni, bi e trifatorial), através de análises fatoriais confirmatórias realizadas com o intuito de testar o ajuste desses modelos teóricos aos dados 
empiricos. Ademais, também foi realizada a avaliação do parâmetro de precisão da medida, através do Alfa de Cronbach e o Ômega de McDonald.

Em relação à validade de construto, o modelo de três dimensões foi corroborado, apresentando índices de ajustes considerados aceitáveis $\left(\chi^{2} / g l=0,89 ; \mathrm{TLI}=, 99 ; \mathrm{CFI}=\right.$,99; RSMEA=0,036). Sobre isso, estudos apontam que em amostras provenientes de populações que apresentam altos índices de dependência, os resultados tendem a apoiar um modelo com um único fator, por outro lado, naquelas onde se verifica um consumo de baixo risco, os resultados apontam uma estrutura com dois e três fatores (Karno, Granholm, \& Lin, 2000; Lima et al., 2005).

Este aspecto é confirmado nesse artigo, uma vez que os dados foram obtidos em uma amostra universitária com baixa prevalência de transtornos relacionados ao consumo bebidas etílicas (74,4\% dos participantes foram classificados na zona de consumo de baixo risco), resultando em uma estrutura de três fatores correspondente ao modelo original desenvolvido pela OMS. Tal resultado foi compatível com estudo realizado numa capital da região nordeste do Brasil. Nesta pesquisa, envolvendo 386 participantes da população geral, os investigadores testaram a adequação do modelo uni e multifatorial do AUDIT. As análises indicaram a adequação da estrutura com três dimensões, com índices de ajustes superiores aos modelos uni e bifatorial, confirmando a estrutura original do instrumento (Santos, Fernandes, Grangeiro, Lopes, \& Sousa, 2013).

Também se mostrou congruente com pesquisa realizada no município de Passos (MG), Brasil, onde foi verificada a invariância do AUDIT em relação a duas formas de aplicação: entrevista pessoal e telefônica. A análise fatorial confirmatória revelou ajustamento do modelo de três fatores aos dados, com índices de ajustes satisfatórios para a entrevista pessoal $\left(\chi^{2} / g l=1,950\right.$; $\mathrm{CFI}=, 928 ; \mathrm{GFI}=, 929 ; \mathrm{RMSEA}=0,078)$ e telefônica $\left(\chi^{2} / g l=1,365 ; \mathrm{CFI}=, 972 ; \mathrm{GFI}=, 950\right.$; RMSEA= 0,049) (Almeida, 2013).

García et al. (2016), em estudo que contou com a participação de 1309 estudantes universitários, também identificaram uma estrutura tridimensional do AUDIT. Contudo, o modelo fatorial obtido não correspondeu exatamente à estrutura original, uma vez que o primeiro fator foi formado pelos itens de 4 a 7, o segundo pelos itens 1, 2, 3 e 8, e o terceiro fator agrupou os itens 9 e 10. Sobre essa variação, pode-se cogitar, conforme Campo-Arias e Oviedo (2008), que esteja relacionada às características da amostra, a forma das perguntas ou a outras variáveis do contexto de aplicaçãodo do instrumento.

No tocante à consistência interna, as estimativas em relação aos escores do AUDIT, utilizando-se o alfa de Cronbach e o ômega de McDonald, podem ser consideradas meritosas, tanto para o fator geral $(\alpha=, 85 ; \Omega=, 89)$ quanto para cada um dos três fatores (Fator 1. $\alpha=, 94 ; \Omega=, 88$; Fator 2. $\alpha=, 81 ; \Omega=, 68$; Fator 3. $\alpha=, 82 ; \Omega=, 66$ ), atestando a adequação do instrumento para a triagem de problemas relativos ao consumo de álcool no ambiente universitário. Esses resultados se coadunam com o que tem sido expresso na literatura especializada, na qual valores, para o alfa e o ômega, superiores a ,70 são considerados satisfatórios (McDonald, 1999; Souza, Alexandre, \& Guirardello, 2017).

A confiabilidade obtida neste estudo para o fator geral, considerando o coeficiente alfa ( $\alpha=$ ,85), é similar à apresentada em pesquisa prévia realizada com uma amostra de homens em Goa, na Índia, na qual o AUDIT demonstrou elevada consistência interna (alfa de Cronbach de ,84; Endsley, Weobong, \& Nadkarni, 2017). Resultado semelhante também foi encontrado em pesquisa desenvolvida com estudantes ingressantes de uma universidade chilena, cuja confiabilidade do instrumento mostrou-se elevada (alfa de ,83) (Seguel Palma et al., 2013), em estudo envolvendo 435 pacientes de um hospital universitário em Chungan, Coreia do sul ( $\alpha=, 88$; Kim et al., 2014), em pesquisa realizada com estudantes mexicanos $(\alpha=, 82$; Gómez-Maqueo, Gómez, Morales, \& Pérez, 2009) e colombianos ( $\alpha=, 77$; Ospina-Díaz et al., 2012).

Em relação ao ômega de McDonald, embora seja pouco informado nas pesquisas acerca da fidedignidade do AUDIT, foram identificados estudos (Arias, Vargas, \& Herazo, 2013; Medina et 
al., 2013) que, de modo equivalente, também relataram este coeficiente, apresentando valores $(\Omega=$ , 87 e $\Omega=, 88$, respectivamente) muito próximos ao obtido neste trabalho $(\Omega=, 89)$, indicando que ômega também é um bom estimador da confiabilidade do AUDIT.

\section{Considerações Finais}

Neste estudo, buscou-se conhecer as propriedades psicométricas do AUDIT em um grupo específico da população (universitários). A partir da comparação entre diferentes modelos (uni, bi e trifatorial), utilizando-se técnicas robustas e de cunho confirmatório, a estrutura trifatorial se destacou como a mais ajustada, com indicadores de ajustes considerados aceitáveis e índices de consistência interna acima dos preconizados pela literatura. Tais aspectos indicam instrumento adequado teórica e metricamente, podendo ser utilizado em pesquisas que objetivam medir padrões de consumo de álcool em amostras similares a aqui considerada.

Contudo, apesar das contribuições desta pesquisa, reconhece-se que a mesma possui limitações. Incialmente, pode-se apontar o viés amostral, já que foi recrutada uma amostra acidental, por conveniência, constituída exclusivamente por estudantes universitários de uma IES pública, o que implica em não representatividade amostral, sendo, portanto, impossível qualquer tentativa de generalização dos resultados apresentados para além da amostra considerada. Outra limitação identificada diz respeito ao instrumento, que sendo de autorrelato, permite que o participante falseie a resposta, em função da desejabilidade social, já que algumas das situações apresentadas nos itens podem ser identificadas como menos socialmente aceitas, enquanto outras são observadas como tabus, o que pode afetar na forma como o item será endossado pelo sujeito (Costa \& Hauck, 2017).

Diante do exposto, e considerando a possibilidade de novos estudos, recomenda-se contar com amostras mais diversificadas desta população (participaram desta pesquisa apenas estudantes de uma IES pública), com o intuito de assegurar maior heterogeneidade, tornando-a representativa. Essa ação é relevante para dirimir possíveis dúvidas acerca da dimensionalidade do AUDIT com esse público-alvo. Também é pertinente contar com pesquisas que possam tentar avaliar evidências psicométricas a partir de outros tipos de validade, a exemplo de validade convergente e discriminante, o que poderia reforçar o corpo de evidências em torno de suas propriedades psicométricas.

Além disso, sugere-se a realização de análises mais específicas, a exemplo das curvas ROC, com valores específicos de sensibilidade e especificidade, apresentando um o ponto de corte mais adequado para as amostras equivalentes à deste estudo, como se identifica em outras pesquisas (Babor et al., 2001; Moretti-Pires \& Corradi-Webster, 2011). Ainda seria pertinente sugerir o controle da desejabilidade, empregando uma medida de desejabilidade social, ou ainda o desenvolvimento de uma medida implícita, com a finalidade de acessar as associações automáticas e espontâneas, que fogem ao controle consciente dos sujeitos (Barbosa, 2015), reduzindo o viés de falseamento das respostas ao instrumento.

\section{Referências}

Adewuya, A. O. (2005). Validation of the alcohol use disorders identification test (AUDIT) as a screening tool for alcohol-related problems among Nigerian university students. Alcohol Alcohol, 40(6), 575-577. doi:10.1093/alcalc/agh197

Allen, J. P., Litten, R. Z., Fertig, J. B., \& Babor, T. (1997). A Review of Research on the Alcohol Use Disorders Identification Test (AUDIT). Alcoholism: Clinical and Experimental Research, 21(4), 613-619. doi:10.1111/j.1530-0277.1997.tb03811.x

Almeida, J. C. (2013). Padrão de consumo de álcool: estudo epidemiológico no município de passos $(M G)$ (Tese de Doutorado). Faculdade de Ciências Farmacêuticas, Universidade Estadual Paulista, Araraquara, SP, Brasil. Recuperado 
dehttps://repositorio.unesp.br/bitstream/handle/11449/100928/000726293.pdf;jsessionid=C A5C0949BB3E36933A9C0F4E22C6B8FB?sequence=1

Altman, D. G., \& Bland, J. M. (1994). Statistics Notes: Diagnostic tests 2: Predictive values. BMJ Clinical Research, 309, 102. doi:10.1136/bmj.309.6947.102

Arias, A., Vargas, M., \& Herazo, E. (2013). Confidencialidad y dimencionalidad del audit en estudiantes de medicina. Psicología desde el Caribe, 30(1), 21-35.

Babor, T. F., Higgins-Biddle, J. C., Saunders, J. B., \& Monteiro, M. G. (2001). The alcohol use disorders identification test. World Health Organization: Geneva.

Baumgarten, L. Z., Gomes, V. L. O., \& Fonseca, A. D. (2012). Consumo alcoólico entre universitários (as) da área da saúde da Universidade Federal do Rio Grande/RS: subsídios para enfermagem. Escola Anna Nery, 16(3), 530-535. doi:10.1590/S141481452012000300015

Cabral, L. R., Farate, C. M. C., \& Duarte, J. C. (2007). Representações Sociais sobre o Álcool em Estudantes do Ensino Superior. Revista referência, 2, 69-80.

Campo-Arias, A., \& Oviedo, H. C. (2008). Propiedades psicométricas de una escala: la consistencia interna. Revistad de Salud Pública, 10(5), 831-839. doi:10.1590/S012400642008000500015

Campos-Arias, A., Villamil-Vargas, M., \& Herazo, E. (2013). Confiabilidad y dimensionalidad del Audit en estudiantes de medicina. Psicología desde el Caribe, 30(1), 21-35.

Cardoso, F. M., Barbosa, H. A., Costa, F. M., Vieira, M. A., \& Caldeira, A. P. (2015). Factors associated with practice of binge drinking among students of health. Revista CEFAC. 17(2), 475-484. doi: 10.1590/1982-021620158914

Carey, K. B., Carey, M. P., \& Chandra, P. S. (2003). Avaliação psicométrica do Teste de Identificação dos Transtornos pelo Uso de Álcool e Teste de Triagem para Abuso de Drogas com pacientes psiquiátricos na Índia. The Journal of Clinical Psychiatry, 64(7), 767-774. doi: 10.4088/JCP.v64n0705

Castañeda, L. T., Munévar, F. R., Lara, L. A. T., \& Villa, Ó. M. G. (2016). Validez y confiabilidad del cuestionario para identificación de trastornos debidos al consumo de alcohol (AUDIT) en universitarios colombianos. Revista Electrónica de Psicología Iztacala, 19(3), 1040-1057.

Chung, T., Colby, S. M., Barnett, N. P., \& Monti, P. M. (2002). Alcohol Use Disorders Identification Test: Factor Structure in an Adolescent Emergency Department Sample. Alcoholism: Clinical and Experimental Research, 26(2), 223-231. doi:10.1111/j.15300277.2002.tb02528.x

Costa, A. R. L., \& Hauck, N, F. (2017). Menos desejabilidade social é mais desejável: Neutralização de instrumentos avaliativos de personalidade. Interação em Psicologia, 21(3), 239-249. doi:10.5380/psiv21i3.53054

Damacena, G. N., Malta, D. C., Boccolini, C. S., Souza, P. R. B., Jr., Almeida, W. D., Ribeiro, L. S., \& Szwarcwald, C. L. (2016). Alcohol abuse and involvement in traffic accidents in the Brazilian population, 2013. Ciência \& Saúde Coletiva, 21(12), 3777-3786. doi: 10.1590/1413812320152112.25692015

Davoren, M. P., Dahly, D., Shiely, F., \& Perry, I. J. (2017). Alcohol consumption among university students: A latent class analysis. Drugs: Education, Prevention and Policy, 25(5), 1-9. doi:10.1080/09687637.2017.1290787

Endsley, P., Weobong, B., \& Nadkarni, A. (2017). The psychometric properties of GHQ for detecting common mental disorder among community dwelling men in Goa, India. Asian Journal Psychiatry, 28, 106-110. doi:10.1016/j.ajp.2017.03.023.

Feijão, I. E. P., Sampaio, H. A. C., Sabry, M. O. D., Carioca, A. A. F., Yum, M. E. M., \& Lima, J. W. O. (2012). Prática de binge alcoólico entre estudantes universitários. Revista Brasileira em Promoção da Saúde, 25(4), 462-468. 
Figlie, N. B., Pillon, S. C., Laranjeira, R., \& Dunn, J. (1997). Does Audit identify a specific for liaison-psychiatric intervention for alcohol dependent patients in a general hospital? Jornal Brasileiro de Psiquiatria, 46, 589-593. doi: 10.1590/S1516-31802000000500005

Gadermann, A. M., Guhn, M., \& Zumbo, B. D. (2012). Estimating ordinal reliability for Likerttype and ordinal item response data: A conceptual, empirical, and practical guide. Practical Assessment, Research \& Evaluation, 17(3), 1-13.

García, C. M., Novalbos, R. J., Martínez, D. J., \& O’Ferrall, G. C. (2016). Validation of the Alcohol Use Disorders Identifi cation Test in university students: AUDIT and AUDIT-C. Adicciones, 28(4), 194-204. doi: 10.20882.775

Gómez-Maqueo, E. L., Gómez, H. H. L., Morales, R. B., \& Pérez, R. M. (2009). Uso del AUDIT y DAST-10 para la identificación del abuso de sustancias psicoactivas y alcohol en adolescentes. Revista Colombiana de Psicología,18(1), 9-17.

Hair, J. F., Black, W. C., Babin, B. J., Anderson, R. E., \& Tatham, R. L. (2009). Análise multivariada de dados. Porto Alegre: Bookman.

Karno, M., Granholm, E., \& Lin, A. (2000). Factor structure of the Alcohol Use Disorders Identification Test (AUDIT) in a mental health clinic sample. Journal of Studies on Alcohol, 61(5), 751-758. doi:10.15288/jsa.2000.61.751

Kim, C.-G., Kim, J. S., Jung, J.-G., Kim, S.-S., Yoon, S.-J., \& Suh, H. -S. (2014). Reliability and validity of Alcohol Use Disorder Identification Test-Korean Revised Version for screening at -risk drinking and alcohol use disorders. Korean Journal of Family Medicine, 35(1), 2-10. doi:10.4082/kjfm.2014.35.1.2

Lima, C. T., Freire, A. C. C., Silva, A. P. B., Teixeira, R., Farrell, M., \& Prince, M. (2005). Concurrent and constructo validity of the AUDIT in an urban Brazilian sample. Alcohol Alcohol, 40(6), 584-589. doi:10.1093/alcalc/agh202

Maciel, M. E. D. (2017). Tradução, adaptação cultural e validação da questão-chave para rastreamento do uso de risco de álcool para o português. (Tese de Doutorado). Escola de Enfermagem do PPGE, Universidade de São Paulo, São Paulo, SP, Brasil.

McDonald, R. P. (1999). Test theory: A unified treatment. Mahwah: Lawrence Erlbaum Associates, Inc.

Medina, A. L., Arévalo, N. M., Beltrán, S. D., Chavarro, Y. L., Herazo, E., \& Campoarias, A. (2013). Consistencia interna y estructura interna del cuestionario AUDIT en amerindios. Investigaciones Andina, 16(28), 871-880.

Mello Jorge, M. H. P., \& Adura, F. E. (2013). Álcool e direção veicular. Revista USP, 96, 23-36. doi:10.11606/issn.2316-9036.v0i96p23-36

Méndez, E. B. (1999). Uma versão brasileira do AUDIT-Alcohol Use Disorders Identification Test. Pelotas: Universidade Federal de Pelotas (Dissertação de Mestrado). Faculdade de Medicina, Universidade Federal de Pelotas, Pelotas, RS, Brasil.

Meneses-Gaya, C. (2011). Estudo de validação de instrumentos de rastreamento para transtornos depressivos, abuso e dependência de álcool e tabaco (Dissertação de mestrado). Faculdade de medicina, Universidade de São Paulo, Ribeirão Preto, SP, Brasil.

Meneses-Gaya, C., Zuardi, A. W., Loureiro, S. R, \& Crippa, J. A. S. (2009). Alcohol Use Disorders Identification Test (AUDIT): an updated systematic review of psychometric properties. Psychology \& Neuroscience, 2(1), 83-97. doi: 10.3922/j.psns.2009.1.12

Moretti-Pires, R. O., Corradi-Webster, C. M. (2011). Adaptation and validation of the Alcohol Use Disorders Identification Test (AUDIT) for a river population in the Brazilian Amazon. Caderno de Saúde Pública. 27(3), 497-509. doi: 10.1590/S0102-311X2011000300010

Noorbakhsh, S., Shams, J., Faghihimohamadi, M., Zahiroddin, H., Hallgren, M., \& Kallmen, H. (2018). Psychometric properties of the Alcohol Use Disorders Identification Test (AUDIT) and prevalence of alcohol use among Iranian psychiatric outpatients. Substance Abuse Treatment, Prevention, and Policy, 13(1), 1-8. doi:10.1186/s13011-018-0141-x 
Ospina-Díaz, J. M., Manrique-Abril, F. G., \& Ariza, N. E. (2012). Confiabillidad y dimensionalidade del Cuestionaario para la Identificación de transtornos relacionados con alcohol (AUDIT) en estudiantes universitários de Tunja (colombia). Salud Uninorte, 28(2), 276-282.

Pérula-de-Torres, L. A., Fernández-García, J. A., Arias-Vega, R., Muriel-Palomino, M., MárquezRebollo, E., \& Ruiz-Moral, R. (2005). Validity of AUDIT test for detection of disorders related with alcohol consumption in women. Medicina Clínica (Barcelona), 125(19), 727 730.

Peuker, A. C., Fogaça, J., \& Bizarro. L. (2006). Expectativas e beber problemático entre universitários. Psicologia Teoria e Pesquisa, 22(2), 193-200. doi: 10.1590/S010237722006000200009

Pinto, J. M. (1999). O grupo na adolescência: vicissitudes do processo de construção/desconstrução. Revista Referência, 2, 27-34.

Rathod, S. D., Nadkarni, A., Bhana, A., \& Shidhaye, R. (2015). Epidemiological features of alcohol use in rural India: a population-based cross-sectional study. BMJ Open, 5(12), 1-9. doi:10.1136/bmjopen-2015-009802

Rocha, L. A., Lopes, A. C. F. M. M., Martelli, D. R. B., Lima, V. B., \& Martelli, H., Jr. (2011). Alcohol use by medical students in Minas Gerais State, Brazil. Revista Brasileira de Educação Médica, 35(3), 369-375. doi:10.1590/S0100-55022011000300010

Santos, W. S., Fernandes, D. P., Grangeiro, A. S. M., Lopes, G., \& Sousa, E. M. P. (2013). Medindo consumo de álcool: análise fatorial confirmatória do Alcohol Use Disorder Identification Test (AUDIT). Psico-USF, 18(1), 121-130.

Santos, W. S., Gouveia, V. V., Fernandes, D. P., Souza, S. S. B., \& Grangeiro, A. S. M. (2012). Alcohol Use Disorders Identification Test (AUDIT): explorando seus parâmetros psicométricos. Jornal Brasileiro de Psiquiatria, 61(3), 117-123. doi:10.1590/S004720852012000300001

Seguel Palma, F., Santander Manríquez, G., \& Alexandre Barriga, O. (2013). Validez y confiabilidad del test de identificación de los trastornos debidos al consumo de alcohol (AUDIT) en estudiantes de una universidad chilena. Ciencia y Enfermería, 19(1), 23-35. doi: $10.4067 / \mathrm{s} 0717-95532013000100003$

Silva, É. C., \& Tucci, A. M. (2014). Estudo transversal sobre o uso de risco de álcool em uma amostra de estudantes de uma universidade federal brasileira. Jornal Brasileiro de Psiquiatria, 63(4), 317-25. doi:10.1590/0047-2085000000040.

Silva, M. F. R., Gomes, A. L. C., Silva, L. R., Braga, J. E. F., Silva, E. F., \& Almeida, E. B. (2015). Relationship between the consumption of alcoholic beverages and academic performance of nursing graduates of a public university. Revista de Pesquisa Cuidado é Fundamental Online, 7, 18-27.

Souza, A. C., Alexandre, N. M. C., \& Guirardello, E B. (2017). Propriedades psicométricas na avaliação de instrumentos: avaliação da confiabilidade e da validade. Epidemiologia e Serviços em Saúde, 26(3), 649-659. doi:10.5123/s1679-49742017000300022

Tabachnick, B. G., \& Fidell, L. S. (2013). Using multivariate statistics. Nova Iorque: Allyn \& Bacon.

Von-der-Pahlen, B., Santtila, P., Witting, K., Varjonen, M., Jern, P., Johansson, A., \& Sandnabba, N. K. (2008). Factor structure of the Alcohol Use Disorders Identification Test (AUDIT) for men and women in different age groups. Journal of Studies on Alcohol and Drugs, 69, 616621.

World Health Organization [Organização Mundial da Saúde, OMS] (2014). Global status report on alcohol and health. Geneva: World Health Organization. 
Participação dos autores: a) Planejamento e concepção do trabalho; b) Coleta de dados; c) Análise e interpretação de dados; d) Redação do manuscrito; e) Revisão crítica do manuscrito.

K.P.A.S. contribuiu em a, c; E.D.M. em b,e; P.C.B.M. em d.

Editora científica responsável: Dra. Cecilia Cracco 